\title{
PDE3A mutations cause autosomal dominant hypertension with brachydactyly
}

\begin{abstract}
Cardiovascular disease is the most common cause of death worldwide, and hypertension is the major risk factor ${ }^{1}$. Mendelian hypertension elucidates mechanisms of blood pressure regulation. Here we report six missense mutations in PDE3A (encoding phosphodiesterase 3A) in six unrelated families with mendelian hypertension and brachydactyly type $E(H T N B)^{2}$. The syndrome features brachydactyly type $E(B D E)$, severe salt-independent but age-dependent hypertension, an increased fibroblast growth rate, neurovascular contact at the rostral-ventrolateral medulla, altered baroreflex blood pressure regulation and death from stroke before age $\mathbf{5 0}$ years when untreated ${ }^{3,4}$. In vitro analyses of mesenchymal stem cell-derived vascular smooth muscle cells (VSMCs) and chondrocytes provided insights into molecular pathogenesis. The mutations increased protein kinase A-mediated PDE3A phosphorylation and resulted in gain of function, with increased cAMP-hydrolytic activity and enhanced cell proliferation. Levels of phosphorylated VASP were diminished, and PTHrP levels were dysregulated. We suggest that the identified PDE3A mutations cause the syndrome. VSMC-expressed PDE3A deserves scrutiny as a therapeutic target for the treatment of hypertension.
\end{abstract}

The mendelian hypertension syndromes described thus far involve increased sodium reabsorption in the distal nephron ${ }^{5}$. The sole exception is autosomal dominant hypertension with BDE (HTNB; MIM 112410 ), first reported in a Turkish kindred ${ }^{2,6}$. HTNB was linked to chromosome $12 \mathrm{p}$ in six unrelated families ${ }^{2,7,8}$. The locus accounts for a difference of $\sim 50 \mathrm{~mm} \mathrm{Hg}$ in mean blood pressure at age 50 years $^{2}$. Penetrance is $100 \%$ (Fig. 1). Previously, we reported a rearrangement on chromosome $12 \mathrm{p}$ shared by all affected families ${ }^{8,9}$. A linkage study in Chinese hypertensive families without BDE identified a linkage signal coinciding with the HTNB locus, supporting the relevance of this locus to essential hypertension ${ }^{10}$.

We performed whole-genome sequencing of Turkish family members and identified a heterozygous missense mutation in PDE3A (NCBI Gene 5139), which encodes a cyclic GMP (cGMP) and AMP (cAMP) phosphodiesterase with a prominent role in the heart, VSMCs, oocytes and platelets ${ }^{11}$. Resequencing of all 48 affected individuals in 6 unrelated families identified 6 independently clustered heterozygous missense mutations in exon 4 of $P D E 3 A$. The altered amino acids, residues 445, 447 and 449, belong to a highly conserved PDE3A domain (Figs. 1 and 2a, Supplementary Fig. 1 and Supplementary Table 1).
We detected none of the previously described chromosomal breakpoints on chromosome 12p12.2-12.1, perhaps owing to high repetitive content in the breakpoint regions (Fig. 2a and Supplementary Table 2) ${ }^{8,9}$. We noted intrafamilial phenotypic variability, with an affected individual (VI/9; 145/77 mm Hg (diastolic/systolic blood pressure) ) showing only mild BDE and height $(159 \mathrm{~cm})$ in the 30th percentile in comparison to one of his severely affected cousins $(142 \mathrm{~cm} \text {, first percentile; VI/1; Fig. 2b })^{7}$. Magnetic resonance imaging (MRI) identified a posterior inferior cerebellar artery (PICA) loop (Supplementary Fig. 2) ${ }^{4}$. Haplotype analysis detected a new recombination event that reduced the size of the linkage interval and eliminated an inversion common to all affected individuals in the six families (Fig. 2c) ${ }^{9}$. In contrast, the affected mother's haplotype showed cosegregation with the more severe brachydactyly phenotype.

PDE proteins are involved during early stages of osteogenesis ${ }^{12}$. PDE4D mutations have been associated with severe brachydactyly in acrodysostosis ${ }^{13,14}$. In mice, $P d e 3 a$ is expressed in the developing limbs, consistent with a role during chondrogenesis (Fig. $2 \mathbf{d}$ and Supplementary Fig. 3a,b). Chondrogenic downregulation of expression of the PTHLH gene, encoding the parathyroid hormone-related peptide PTHrP, was associated with $\mathrm{BDE}^{15}$. We also observed PTHLH downregulation in chondrogenically induced fibroblasts from affected individuals (Fig. 2e and Supplementary Fig. 3c).

We addressed the functional consequences of the identified PDE3A mutations in HeLa cells expressing the mutant proteins. Forskolin and L-arginine stimulate adenylate and guanylate cyclases, respectively, to enhance cellular cAMP and cGMP levels ${ }^{16,17}$. We detected significantly reduced cAMP levels in comparison to wild-type PDE3A $(P \leq 0.001)$, consistent with gain-of-function mutations, with no change in cGMP levels (Supplementary Fig. 4a,b). Three PDE3A isoforms-PDE3A1 (microsomal), PDE3A2 and PDE3A3 (both microsomal and cytosolic) - have been identified in human myocardium ${ }^{18,19}$. PDE3A3 does not contain the sequence harboring the altered residues. The predominant isoform in VSMCs is PDE3A2 (refs. 18,20). To directly elucidate the effects of the mutations, we compared the Michaelis-Menten kinetics of cAMP-hydrolytic activity for recombinant Flag-tagged wild-type and Thr445Asn PDE3A1 and for wild-type and Thr445Asn PDE3A2 purified from transfected cells (Fig. 3a,b and Supplementary Fig. 4d-k). The p.Thr445Asn alteration increased the affinity (decreased the $K_{\mathrm{M}}$ ) of both PDE3A1 and PDE3A2 for cAMP without affecting $V_{\max }$ (the maximum catalytic velocity of the substrate-saturated enzyme). This increase in affinity relative to the wild-type enzyme was enhanced for both isoforms

A full list of authors and affiliations appears at the end of the paper. 


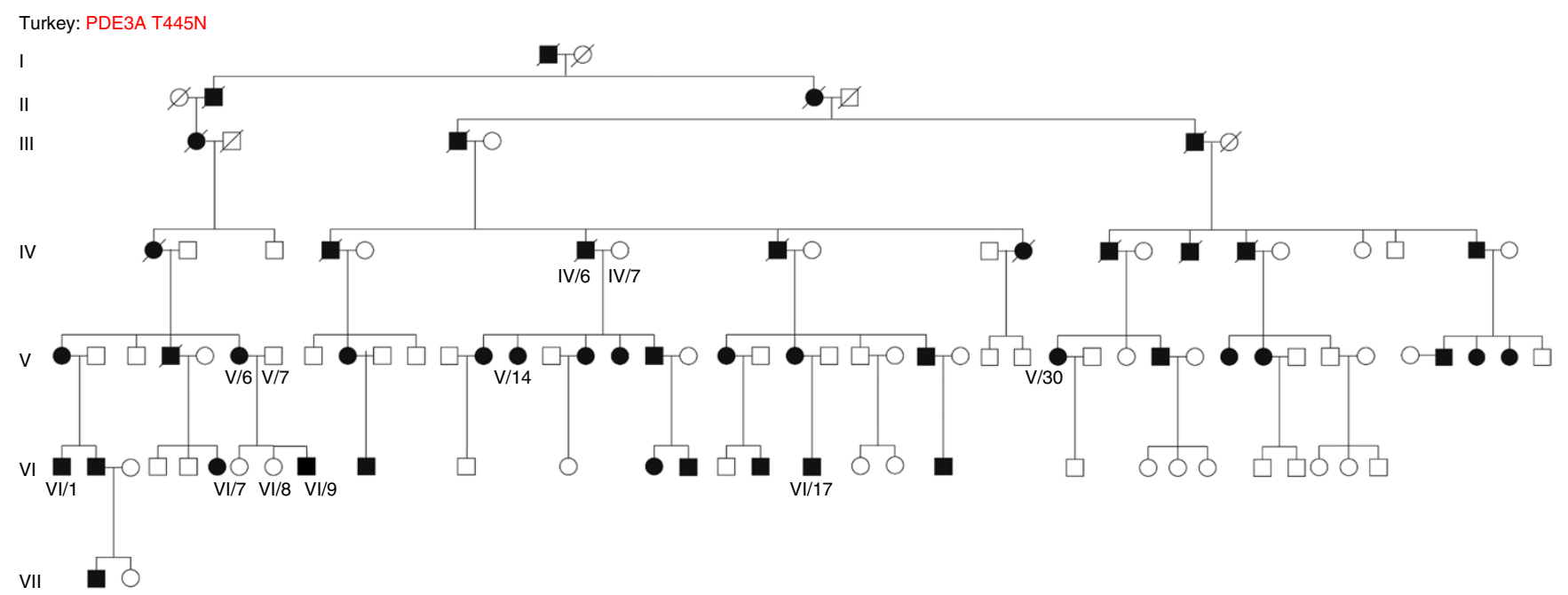

France: PDE3A T445A

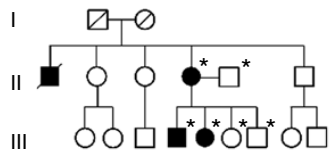

United States: PDE3A T445S

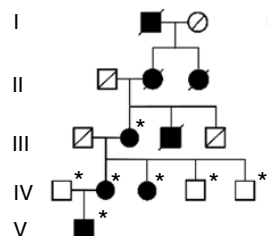

South Africa: PDE3A A447T

I $\square{ }^{*} \mathrm{O}^{*}$

II $0^{*} \mathrm{O}^{*}$

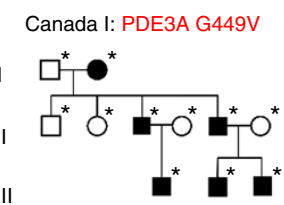

Canada II: PDE3A A447V

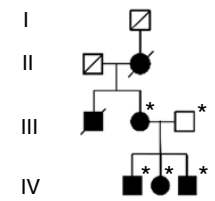

Figure 1 Pedigrees and PDE3A missense mutations in six unrelated families with autosomal dominant hypertension with BDE (HTNB). The genomes of the Turkish family members IV/6, IV/7, V/14 and V/30 were sequenced. DNA from all affected and unaffected Turkish family members was used for Sanger sequencing of PDE3A. The asterisks in the pedigrees of the other families indicate PDE3A resequencing of family members. In all affected individuals (black symbols), a PDE3A mutation was found, in contrast to in their unaffected, related family members (white symbols), demonstrating complete penetrance in all families. The nuclear family comprising V/6, V/7 and VI/7-VI/9 was used for the haplotype analysis shown in Figure 2c. $\mathrm{X}$-rays of $\mathrm{VI} / 1$ and $\mathrm{VI} / 9$ are shown in Figure $2 \mathrm{~b}$. MSCs were extracted from VI/9 and $\mathrm{VI} / 17$.

when CAMP-mediated signaling was stimulated by exposure of the transfected cells to forskolin. Half-maximal inhibitory concentration $\left(\mathrm{IC}_{50}\right)$ measurements for milrinone, a PDE3 inhibitor used to augment cardiac contractility and decrease vascular resistance in patients with heart failure ${ }^{21,22}$, showed that higher concentrations of the inhibitor were necessary to inhibit the recombinant Thr445Asn forms of both PDE3A1 and PDE3A2 (Fig. 3c,d), whereas the $\mathrm{IC}_{50}$ values for $\mathrm{cGMP}$ inhibition of cAMP-hydrolytic activity were nearly equivalent for the wild-type and mutant isoforms (Supplementary Fig. 5).

We also used a vector with the luciferase gene under the control of a cAMP-responsive element (CRE) to determine the effects of all six PDE3A mutations on CAMP-mediated signaling in cells ${ }^{23}$. Consistent with the observed gain-of-function effects from increased cAMP-hydrolytic activity, the expression of all six PDE3A mutants decreased luciferase expression in comparison to wild-type PDE3A (Supplementary Fig. 6).

We obtained mesenchymal stem cells (MSCs) from two affected individuals with the p.Thr445Asn alteration (VI/9 and VI/17). MSCs were characterized by FACS and differentiated into adipocytes, osteocytes and chondrocytes, demonstrating their multilineage potential (Supplementary Fig. 7a,b) ${ }^{24}$. MSC-derived VSMCs expressed smooth muscle actin $\alpha$ (SMA $\alpha$ ), calponin and transgelin at high levels (SM22 $\alpha$; Supplementary Fig. 7c). Deletion of mouse Pde $3 a$ suppressed VSMC proliferation, indicating the importance of PDE3A during arteriogenesis and arterial remodeling ${ }^{25}$. Therefore, we focused on the vascular relevance of the $P D E 3 A$ mutations. In our earlier studies, we had observed an enhanced cell growth rate for fibroblasts and hyperplastic arterial walls from affected individuals, concerning the thickened
VSMC layer ${ }^{9,26}$. We hypothesized that the PDE3A mutations were responsible for enhanced mitosis and arterial remodeling. In HeLa cell proliferation assays with full-length $P D E 3 A$ transfection constructs (encoding PDE3A1 and PDE3A2), as well as in VSMCs derived from patients with the p.Thr445Asn substitution, we observed higher mitotic rates in cells expressing mutant PDE3A. Transfection with vector for wild-type PDE3A also increased cell growth in comparison to endogenous levels of PDE3A (Fig. 3e,f and Supplementary Fig. 8).

The Ser428 and Ser438 residues that are adjacent to the residues altered by the $P D E 3 A$ mutations can be phosphorylated by protein kinase $\mathrm{C}$ (PKC) or protein kinase A (PKA), causing increased cAMP hydrolysis (Fig. 2a) ${ }^{18,27,28}$. Thus, we investigated phosphorylated PDE3A levels without and after exposure to PMA (phorbol-12myristate-13-acetate; to activate $\mathrm{PKC}$ ) or forskolin (to increase cAMP content and thereby activate PKA) ${ }^{29,30}$. Phosphorylation of PDE3A1 Thr445Asn and PDE3A2 Thr445Asn at Ser428 and Ser438 was increased in PMA- and forskolin-treated transfected HeLa cells, in comparison to cells with endogenous protein levels or cells transfected to express wild-type PDE3A (Fig. 4a-e and Supplementary Fig. 9) ${ }^{19}$. Furthermore, in unstimulated MSCs and VSMCs derived from the affected individuals harboring p.Thr445Asn, we detected higher PDE3A Ser428 and Ser438 phosphorylation levels (Fig. 4f,g). Inhibiting PKA or PKC showed that both kinases phosphorylated PDE3A Ser438 (Supplementary Fig. 10). We investigated whether the alteration in PDE3A phosphorylation could be attributed to preferential mRNA expression from the mutated allele. We used pyrosequencing to dissect allele usage and detected no differences between the wild-type and mutated alleles (Supplementary Fig. 11). 
a hg19, chr. 12 (p12.2-12.1)

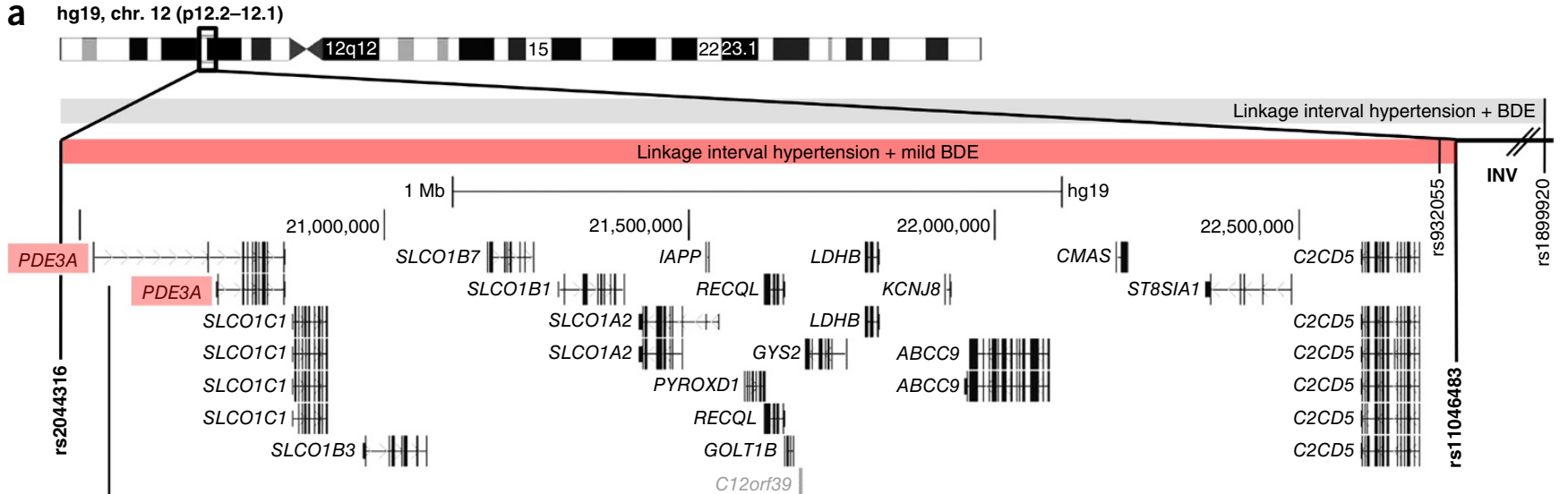

\begin{tabular}{|c|c|c|c|c|c|c|c|c|}
\hline IPK & $\begin{array}{c}428 \\
\text { RLRRS }\end{array}$ & $\begin{array}{c}445 \\
T \\
\text { ACC }\end{array}$ & $\begin{array}{c}\mathrm{S} \\
\mathrm{TCG}\end{array}$ & $\begin{array}{c}447 \\
\text { A } \\
\text { GCC }\end{array}$ & $\begin{array}{c}\mathrm{T} \\
\mathrm{ACA}\end{array}$ & $\begin{array}{c}449 \\
\text { G } \\
\text { GGT }\end{array}$ & ${ }^{450}$ LPTLEPAPVRRDRSTSIKLQEAPSSS & $3^{\prime}$ \\
\hline
\end{tabular}

b

$\mathrm{VI} / 1$, affected 2,15 years

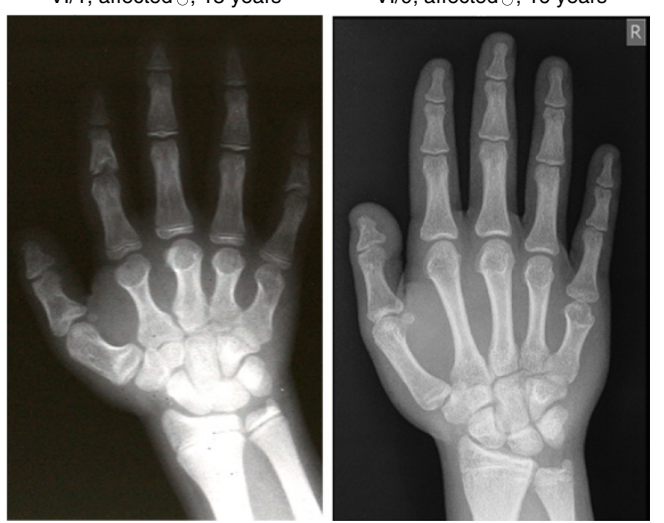

C

rs 10505879 rs10842031 rs932055 rs11046483 rs11046677

rs10505879 rs10842031 rs932055 rs11046483 rs11046677

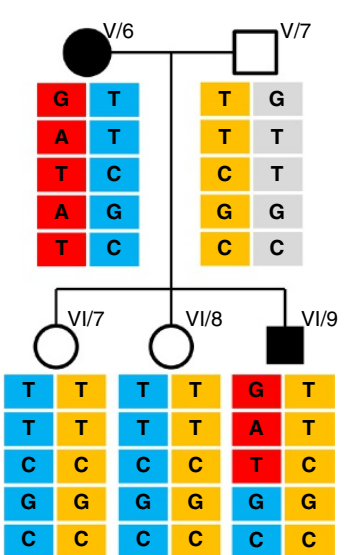

d

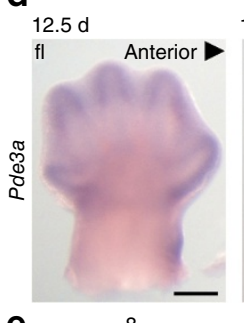

e

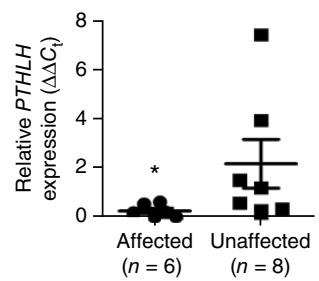

Figure 2 The HTNB linkage interval and expression studies. (a) The linkage interval of all families (gray bar) with flanking SNPs on chromosome 12p12.2-12.1 and the previously described inversion (INV) is shown (human genome assembly hg19). The red bar shows the linkage interval of a hypertensive boy with mild BDE and nearly average height by age $(\mathrm{VI} / 9 ; \mathrm{c})$ with all annotated RefSeq genes. The peptide sequence encoded by PDE3A exon 4 that harbors the mutations (which alter amino acids 445, 447 and 449) and the phosphorylation sites Ser428 and Ser438 near the altered residues are shown. The PDE3A Ile421-Leu450 sequence (30-mer peptide) was used for peptide SPOT assays (Fig. 5 and Supplementary Figs. 13 and 14). (b) Mild BDE of VI/9 and much more severe BDE of his cousin, VI/1. (c) Haplotype analysis of the Turkish nuclear family identified a new recombination event excluding the inversion from linkage in VI/9 (black square), in comparison to his mother, V/6 (black circle). (d) Chondrogenic Pde3a expression in developing mice at 12.5 and $13.5 \mathrm{~d}$ was studied by RNA in situ hybridization. Pde3a was expressed in domains involved in digit formation. Scale bars, $1 \mathrm{~mm}$. (e) BDE-associated PTHLH expression was downregulated in chondrogenically induced fibroblasts from six age- and sexmatched affected individuals versus eight unaffected controls (mean \pm s.e.m., Wilcoxon-Mann-Whitney rank-sum test, ${ }^{*} P=0.022$ ).

Reduced phosphorylation of vasodilator-stimulated phosphoprotein (VASP) at Ser157, mediated by PKA, has been linked to enhanced VSMC proliferation after angioplasty ${ }^{31}$. Elevated full-length PTHrP levels enhance neointimal proliferation, whereas the PTHrP peptide (residues 1-36) inhibits VSMC proliferation ${ }^{31,32}$. We observed reduced VASP Ser 157 phosphorylation and elevated PTHrP levels in PMA-stimulated HeLa cells expressing the mutant enzymes. VASP Ser 157 phosphorylation was not greatly altered in forskolin-treated HeLa cells expressing the mutant enzymes, presumably because the forskolin-induced increase in cAMP levels was sufficient for PKA activation despite hyperactive PDE3A (Fig. 4h). Moreover, we detected increased expression of full-length PTHrP protein and decreased expression of the PTHrP peptide (residues 1-36) in cells from affected individuals (Supplementary Fig. 12).

In a peptide SPOT assay, we synthesized 30-mer peptides representing Ile421-Leu450 of wild-type PDE3A and the six mutants, including alanine or aspartic acid replacements for Ser428 and Ser438 (Figs. 2a and 5a, and Supplementary Fig. 13) ${ }^{33}$. The spotting of Ser438 or prephosphorylated Ser428 or Ser438, followed by in vitro phosphorylation by PKA, identified significantly enhanced phosphorylation at the PDE3A Ser438 residue of the six mutants, confirming the previous results (Fig. 5a,b and Supplementary Fig. 14a-c). To further elucidate the increased phosphorylation signal at Ser438 (peptide lanes 7 and 9), we examined whether PKA phosphorylated additional residues. Interestingly, the PKA signals increased even when Ser428 and Ser 438 were substituted with alanine residues. This result could be due to increased levels of phosphorylation at Ser439 and Thr440, which are close to Ser438 (Supplementary Fig. 14d-f).

Finally, we replaced Ser428, Ser438, Ser439 and Thr440 with alanine residues and repeated the Michaelis-Menten kinetic analysis of Flag-tagged wild-type PDE3A1 and PDE3A2 versus the Thr445Asn mutant isoforms. The alanine substitutions diminished cAMP 
a
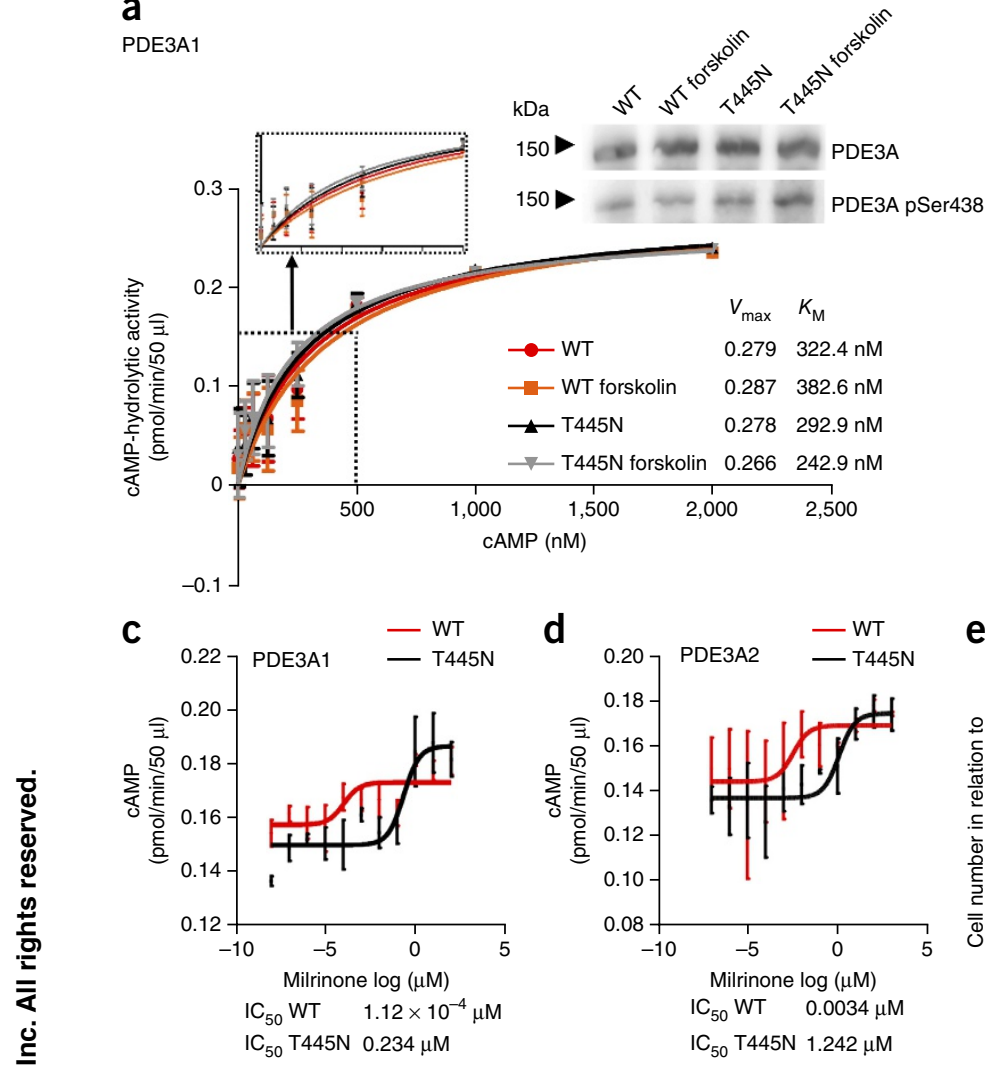
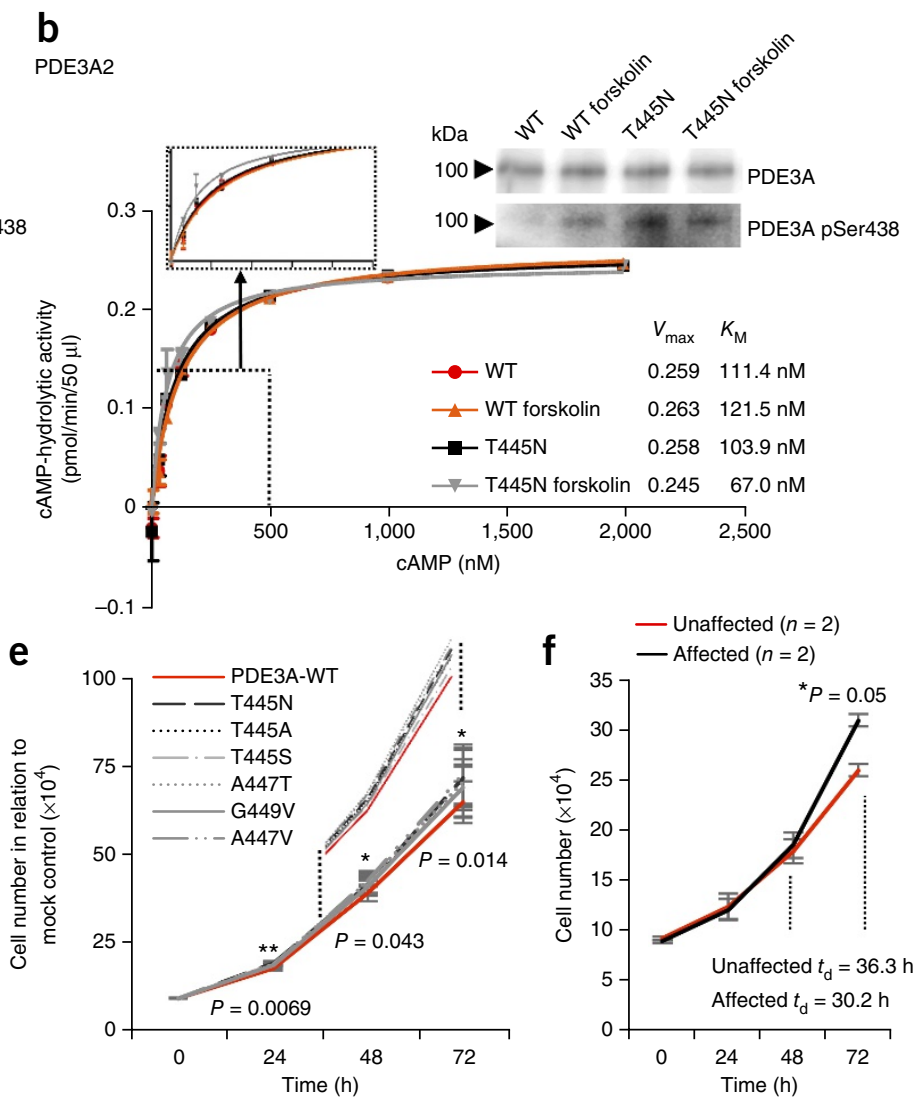

Figure 3 Michaelis-Menten kinetics, IC 50 measurements and CFSE proliferation assays. HeLa cells were transiently transfected to express Flag-tagged wild-type (WT) PDE3A or PDE3A with the p.Thr445Asn alteration. The recombinant PDE3A1 and PDE3A2 proteins were purified via their Flag tags. (a,b) In Michaelis-Menten kinetic analysis of PDE3A1 (a) and PDE3A2 (b), $V_{\max }$ and $K_{M}$ values showed that the Thr445Asn mutant purified from unstimulated and forskolin-stimulated cells $(20 \mu \mathrm{M}$ forskolin for $30 \mathrm{~min})$ had increased cAMP affinity in comparison to wild-type PDE3A (mean \pm s.e.m.; $n=3$ ). pSer438, phosphorylation at Ser438. (c) For the milrinone inhibitor, the IC 50 value for wild-type PDE3A1 was $1.12 \times 10^{-4} \mu \mathrm{M}$, in contrast to $0.234 \mu \mathrm{M}$ for the Thr445Asn mutant $(n=3)$. (d) For wild-type PDE3A2, the IC 50 for milrinone was $0.0034 \mu \mathrm{M}$, in comparison to $1.242 \mu \mathrm{M}$ for PDE3A2 Thr445Asn $(n=2)$. (e) A proliferation assay showed an increased mitosis rate with all six PDE3A mutants in comparison to wild-type PDE3A after expression in HeLa cells (mean \pm s.e.m.; $n=3$; normalized to the endogenous PDE3A levels of mock-transfected control; Wilcoxon-Mann-Whitney rank-sum test, ${ }^{* *} P<0.01,{ }^{*} P<0.05$ ). The difference in cell numbers between cells expressing mutant and wild-type PDE3A was a modest $6.2 \%$ with a maximum of $9.5 \%$. (f) Proliferation assay of two patient-derived VSMC lineages (affected) and two control lineages (unaffected). The patient-derived VSMCs proliferated faster than the controls: the doubling time $\left(t_{\mathrm{d}}\right)$ for control cells was $36.3 \mathrm{~h}$ and was $30.2 \mathrm{~h}$ for patient-derived cells (mean \pm s.e.m.; $n=3$; Wilcoxon-Mann-Whitney test, $\left.{ }^{*} P=0.05\right)$. The results for the functional assays in this figure are from biological replicates.

hydrolysis, supporting the notion that mutation-dependent enhanced phosphorylation at these sites is responsible for increased cAMP hydrolysis (Fig. 5c).

We observed that mutant PDE3A was more extensively phosphorylated than wild-type PDE3A at the Ser428 and Ser438 residues and that PTHrP isoforms were dysregulated. Our results suggest that increased PKA-mediated phosphorylation at Ser438 and PKC-mediated phosphorylation at Ser428, reported to increase PDE3A2 activity ${ }^{27}$, may have contributed to the increase in the cAMP-hydrolytic activity of the mutant enzymes, leading to reduced cAMP levels and enhanced cell proliferation. This result would be consistent with the increased affinity of the Thr445Asn mutants for cAMP that we observed after exposure of transfected cells to forskolin (Fig. 3a,b). Further studies will aim to characterize the possible allosteric effects of these mutations on other phosphorylation sites that may modulate enzyme activity and proteinprotein interactions involved in PDE3A function-for example, the interaction of PDE3A with phospholamban, SERCA2 and AKAP18 described in mouse and human heart ${ }^{34-36}$.

Augmented full-length PTHrP levels, decreased amounts of PTHrP peptide (residues 1-36) and reduced VASP Ser157 phosphorylation may additionally promote VSMC proliferation in individuals with these gain-of-function $P D E 3 A$ mutations, leading to vessel wall hyperplasia. The $\mathrm{IC}_{50}$ measurements showed that a higher milrinone concentration is necessary to abrogate the increased hydrolysis of cAMP by PDE3A1 Thr445Asn and PDE3A2 Thr445Asn. These data are compatible with our previous results indicating that milrinone-induced forearm vasodilation was not significantly different in affected individuals and controls ${ }^{8}$.

Our data strongly suggest that PDE3A mutations in HTNB are responsible for hypertension by contributing to a general increase in peripheral vascular resistance (Supplementary Fig. 15) ${ }^{2,26}$. The fact that PDE3 inhibition lowers blood pressure in patients with hypertensive heart failure and in patients with pulmonary hypertension is consistent with this hypothesis ${ }^{37}$. cAMP inhibits myosin light chain kinase (MLCK) through phosphorylation of the latter by PKA, thereby causing VSMC relaxation. Dephosphorylation of myosin light chains by MLC phosphatase also produces relaxation ${ }^{38}$. Enhanced PDE3A activity could, by lowering the intracellular cAMP concentration in VSMCs, promote increased peripheral vascular constriction. Furthermore, increased VSMC proliferation could induce remodeling 
a

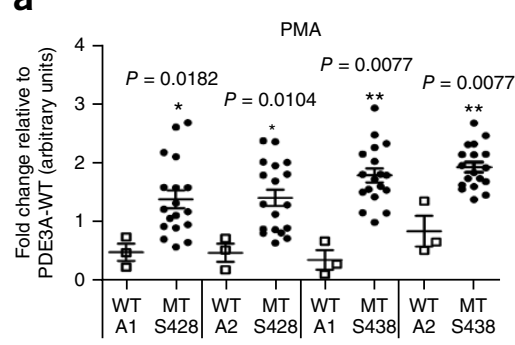

b

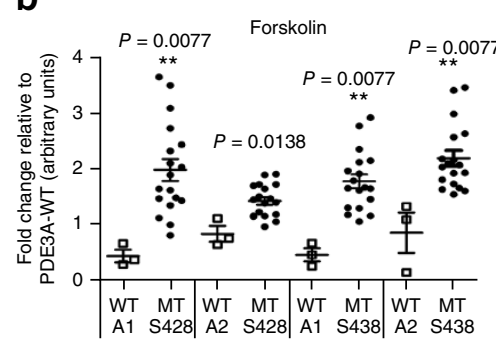

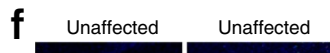
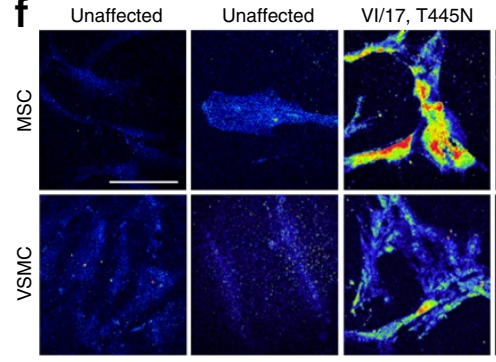

$\mathrm{VI} / 9, \mathrm{~T} 445 \mathrm{~N}$

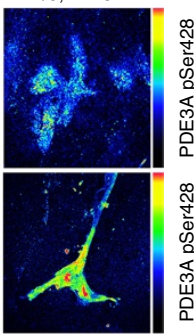

g Unaffected

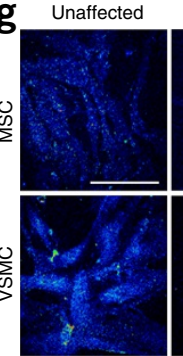

Unaffected

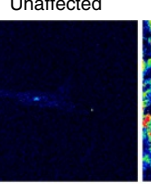

$\mathrm{V} / 177, \mathrm{~T} 445 \mathrm{~N}$

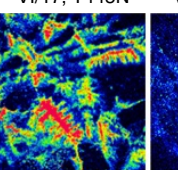

$\mathrm{Vl} / 9, \mathrm{~T} 445 \mathrm{~N}$

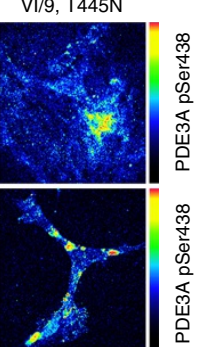

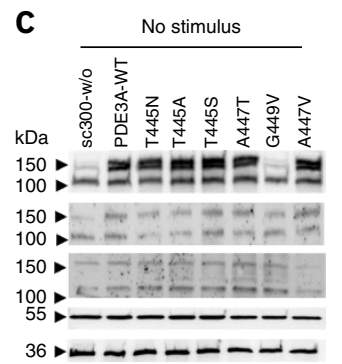

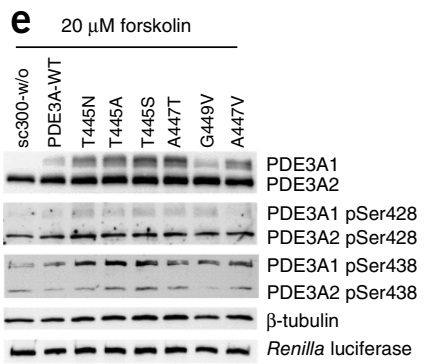

h

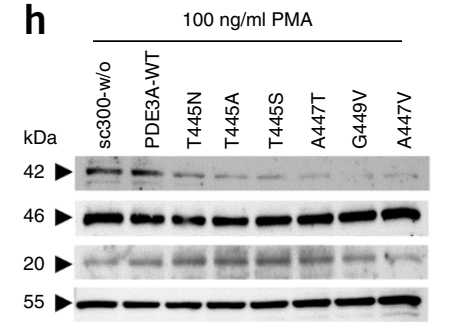

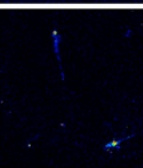

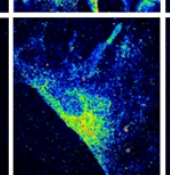

$20 \mu \mathrm{M}$ forskolin

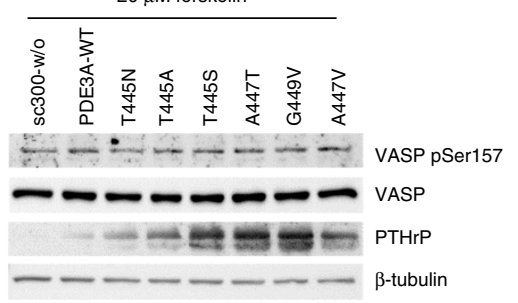

Figure 4 PDE3A Ser428 and Ser438 phosphorylation in HeLa cells, MSCs and MSC-derived VSMCs. (a,b) In densitometric immunoblotting quantification of PMA-stimulated $(100 \mathrm{ng} / \mathrm{ml}$ PMA for $30 \mathrm{~min}$ ) (a) and forskolin-treated ( $20 \mu \mathrm{M}$ forskolin for $30 \mathrm{~min}$ ) (b) HeLa cells, significant differences in phosphorylation between cells transfected to express wild-type PDE3A or each mutant (MT) PDE3A enzyme were determined at Ser428 and Ser438 after normalization to $\beta$-tubulin levels. Differences in signal are shown as fold change relative to the signal observed with expression of transfected wild-type PDE3A (mean \pm s.e.m.; $n=3$; two-tailed Wilcoxon-Mann-Whitney test, ${ }^{* *} P<0.01,{ }^{*} P<0.05$ ). (c-e) HeLa cells transiently expressing the six PDE3A mutants were unstimulated (c), stimulated with PMA (d) or treated with forskolin (e). Equivalent expression of cotransfected Renilla luciferase (pRL-TK) confirmed equal transfection conditions; endogenous $\beta$-tubulin was used as a loading control. The antibody to PDE3A detects the PDE3A1 and PDE3A2 isoforms. Experimental and variation differences in phosphorylation were detected upon normalization with the loading control and are summarized in $\mathbf{a}$ and $\mathbf{b}$ (individual quantification values are given in Supplementary Fig. 9) $(n=3)$. Control was empty vector (sc300-w/o). (f,g) Differences in phosphorylation of PDE3A at Ser428 (f) and Ser438 (g) were documented for two unaffected controls relative to two affected individuals using semiquantitative immunofluorescence. The pseudocolor spectrum indicates signal intensities from low (black) to high (red). PDE3A was highly phosphorylated in the affected cells expressing the Thr445Asn mutant in comparison to the control cells, for MSCs and VSMCs. Scale bars, $50 \mu \mathrm{m}$. (h) PMA stimulation caused reduced PKA-mediated VASP Ser157 phosphorylation and higher PTHrP expression in cells expressing the PDE3A mutants relative to controls. The expression of non-phosphorylated VASP was not different.

favoring hypertension, perhaps by changes in vascular structure or neurovascular contact. Our studies of PDE3A mutants with increased activity showing enhanced mitosis are consistent with the observed decrease in VSMC proliferation when Pde3a was deleted in an earlier study ${ }^{25}$.

The milder BDE of subject VI/9 suggests that regulatory DNA elements or noncoding RNAs in the excluded inversion region epigenetically trigger the pathogenesis of $\mathrm{BDE}$ and short stature. We found that PDE3A, which is involved in mouse chondrogenesis, could contribute to $P$ THLH downregulation, which has been associated with $\mathrm{BDE}^{15,39}$. In chondrocytes, PTHrP is regulated by cAMP and transduces signals through the $\mathrm{PTH} / \mathrm{PTH} \mathrm{P}$ receptor, activating adenylate cyclase for cAMP production ${ }^{40,41}$. We suggest that increased cAMP hydrolysis could cause PTHrP downregulation through a regulatory feedback loop (Supplementary Fig. 15).

In conclusion, we have identified independently clustered $P D E 3 A$ mutations in individuals with HTNB, which exhibit a gain-of-function effect on cAMP hydrolysis. We found evidence for mechanisms mediating VSMC hyperplasia and increased peripheral vascular resistance. Studying the functional consequences of the PDE3A mutations in animal models will result in further insight into normal and pathogenic vascular and skeletal development.

\section{METHODS}

Methods and any associated references are available in the online version of the paper.

Note: Any Supplementary Information and Source Data files are available in the online version of the paper.

\section{ACKNOWLEDGMENTS}

We thank all family members for their cooperation. We thank M.-B. Köhler and M. Toliat for technical assistance. P.G.M., F.C.L., O.T. and S.B. received support from the Deutsche Forschungsgemeinschaft (DFG; BA1773/4-1, BA1773/4-2, MA5028/1-2 and MA5028/1-3) and grants-in-aid from the German Hypertension Society (Deutsche Hochdruckliga, DHL) and from the German Heart Research Foundation (F/24/13). E.K. was supported by the DFG (KL1415/4-2), the Else Kröner-FreseniusStiftung (2013_A145) and the German-Israeli Foundation (I-1210-286.13/2012). F.C.L. received support from the Lingen-Stiftung. F.V. and M.A.M. were supported by the US Department of Veterans Affairs (CARA-029-09F), the American Heart Association (10034439) and the University of Utah Research Foundation. James C. Melby referred one of the families. Dr. Melby died on 19 August 2007.

\section{AUTHOR CONTRIBUTIONS}

N.B. first described this syndrome in 1973. F.C.L. and his laboratory have pursued this project since 1994. O.T., H.R.T., H. Schuster, J.J., J.T., H.H., R.H., L.O.H. and R.N. phenotyped the syndrome. D.C., M.G.B., G.P., M.H. and H.R.T. identified additional families with the syndrome. T.F.W., J.O., S.B., A.B. and F.R. performed microsatellite and SNP linkage analyses. M.G. and N.H. performed genotyping 

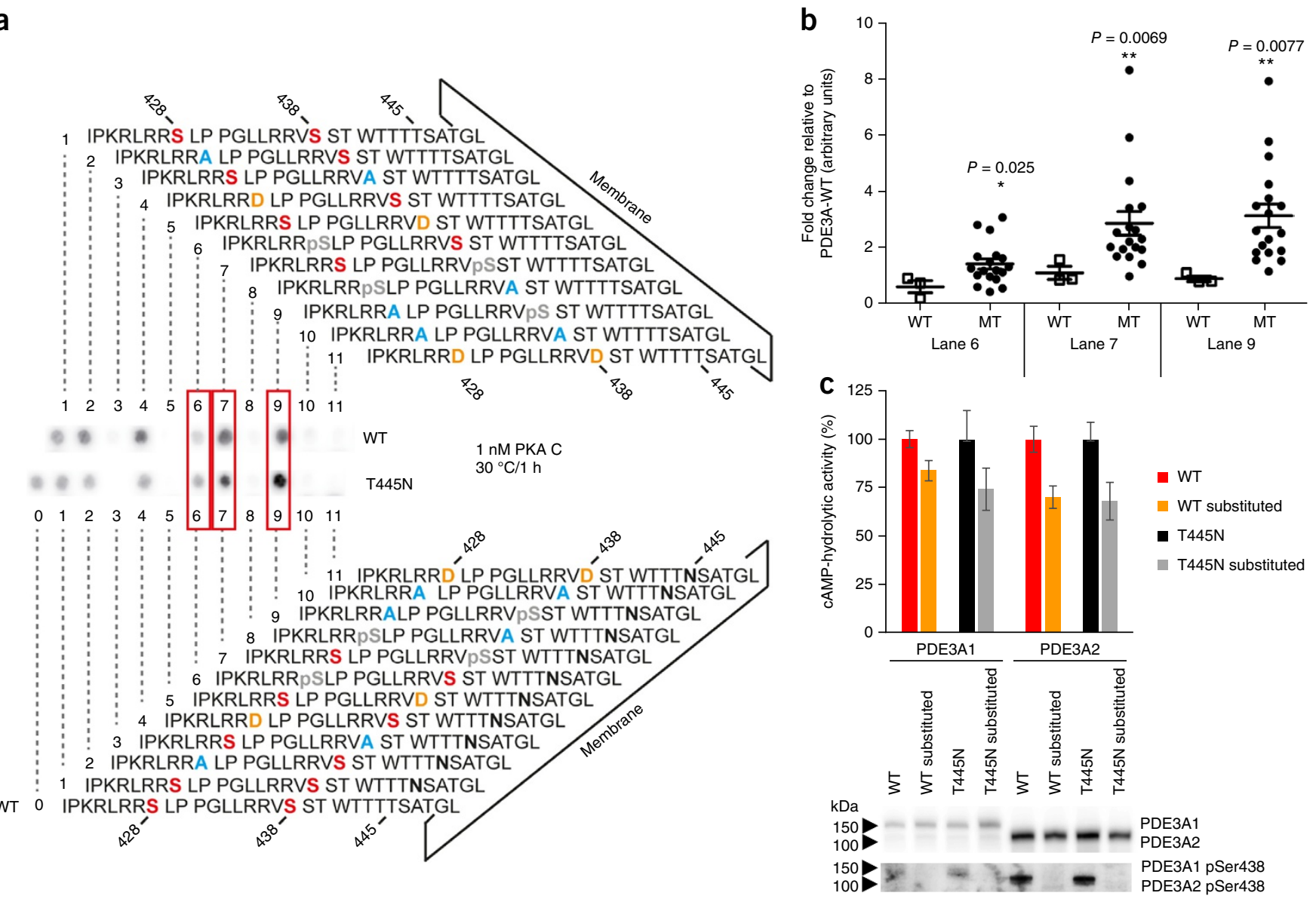

Figure 5 Peptide SPOT assay of the PDE3A Thr445Asn mutant. (a) Wild-type PDE3A and PDE3A Thr445Asn mutant peptides representing Ile421Leu450 were spot synthesized (sequences in Supplementary Fig. 13a). Ser428 and Ser438 were replaced by alanine and aspartic acid residues, as indicated. For visual simplicity, signals for wild-type PDE3A versus the PDE3A Thr445Asn mutants are shown (peptide spots for the remaining mutants are shown in Supplementary Fig. 13b). PKA phosphorylation of peptides with prephosphorylated Ser428 resulted in higher Ser438 phosphorylation of the Thr445Asn mutant (lane 6). Prephosphorylated Ser438 and subsequent incubation with PKA also caused enhanced phosphorylation signals for all PDE3A mutants (lanes 7 and 9; Supplementary Figs. 13b and 14b,c). (b) Densitometric quantification of the peptide spots for all mutants. The signals for lanes 6, 7 and 9 showed significantly enhanced phosphorylation after incubation with PKA (mean \pm s.e.m.; biological replicates, $n=3$; two-tailed Wilcoxon-Mann-Whitney rank-sum test, $\left.{ }^{*} P<0.01,{ }^{*} P<0.05\right)$. (c) Michaelis-Menten kinetics of Flag-tagged wild-type PDE3A1, wild-type PDE3A2, PDE3A1 Thr445Asn and PDE3A2 Thr445Asn in comparison to Flag-tagged PDE3A isoforms with alanine substitutions at Ser428, Ser438, Ser439 and Thr440. The alanine substitutions led to a decrease in cAMP hydrolysis (mean \pm s.d.; $n=3$ biological replicates). The data shown are the ratios of phosphorylated wild-type PDE3A or PDE3A Thr445Asn to the corresponding alanine-substituted isoforms. Immunoblotting verified equal PDE3A amounts during the functional Michaelis-Menten kinetics measurements and diminished Ser438 phosphorylation with the alanine substitutions.

analyses within the families and also analyzed Chinese hypertensive families that showed linkage to the chromosome 12p locus. A.W., M.K., A.R., K.R. and T.L. performed cytogenetics. S.S. performed in situ mouse studies. S.M., P.M.K., D.P. and J.H. carried out Illumina whole-genome sequencing. A.A., P.G.M. and S.B. analyzed Complete Genomics whole-genome sequencing data, and A.A. identified the PDE3A mutation. H. Schulz statistically analyzed various data. C.L. and A.A. performed the confocal immunofluorescence imaging. F.Q., I.H., E.B.-K. and A.M. performed technical studies. K.M. and Y.W.-N. prepared MSCs. M.V. kindly provided unaffected MSCs and supported all the MSC investigations. Y.W.-N., A.A. and P.G.M. analyzed cell proliferation. F.V. and M.A.M. provided Flag-tagged PDE3A expression constructs and provided intellectual input. C.S. and E.K. performed ELISA assays on recombinant proteins and peptide SPOT assays. P.G.M. participated in all scientific aspects of the study and was personally responsible for the PDE3A functional assays, $\mathrm{IC}_{50}$ determinations and work with MSCs. P.G.M., F.C.L. and S.B. wrote the manuscript. The manuscript was the product of more than 20 years of research to which all authors have contributed.

\section{COMPETING FINANCIAL INTERESTS}

The authors declare no competing financial interests.

Reprints and permissions information is available online at http://www.nature.com/ reprints/index.html.
1. Hunter, D.J. \& Reddy, K.S. Noncommunicable diseases. N. Engl. J. Med. 369 1336-1343 (2013)

2. Schuster, H. et al. Severe autosomal dominant hypertension and brachydactyly in a unique Turkish kindred maps to human chromosome 12. Nat. Genet. 13, 98-100 (1996).

3. Schuster, $\mathrm{H}$. et al. A cross-over medication trial for patients with autosomaldominant hypertension with brachydactyly. Kidney Int. 53, 167-172 (1998).

4. Naraghi, R. et al. Neurovascular compression at the ventrolateral medulla in autosomal dominant hypertension and brachydactyly. Stroke 28, 1749-1754 (1997).

5. Lifton, R.P. Genetic dissection of human blood pressure variation: common pathways from rare phenotypes. Harvey Lect. 100, 71-101 (2004).

6. Bilginturan, N., Zileli, S., Karacadag, S. \& Pirnar, T. Hereditary brachydactyly associated with hypertension. J. Med. Genet. 10, 253-259 (1973).

7. Toka, O. et al. Childhood hypertension in autosomal-dominant hypertension with brachydactyly. Hypertension 56, 988-994 (2010).

8. Bähring, S. et al. Autosomal-dominant hypertension with type E brachydactyly is caused by rearrangement on the short arm of chromosome 12. Hypertension $\mathbf{4 3}$ 471-476 (2004).

9. Bähring, S. et al. Inversion region for hypertension and brachydactyly on chromosome $12 p$ features multiple splicing and noncoding RNA. Hypertension 51, 426-431 (2008).

10. Gong, M. et al. Genome-wide linkage reveals a locus for human essential (primary) hypertension on chromosome 12p. Hum. Mol. Genet. 12, 1273-1277 (2003).

11. Maurice, D.H. et al. Cyclic nucleotide phosphodiesterase activity, expression, and targeting in cells of the cardiovascular system. Mol. Pharmacol. 64, 533-546 (2003). 
12. Wakabayashi, S. et al. Involvement of phosphodiesterase isozymes in osteoblastic differentiation. J. Bone Miner. Res. 17, 249-256 (2002)

13. Michot, $C$. et al. Exome sequencing identifies $P D E 4 D$ mutations as another cause of acrodysostosis. Am. J. Hum. Genet. 90, 740-745 (2012).

14. Lee, $\mathrm{H}$. et al. Exome sequencing identifies PDE $4 D$ mutations in acrodysostosis. Am. J. Hum. Genet. 90, 746-751 (2012).

15. Maass, P.G. et al. A misplaced IncRNA causes brachydactyly in humans. J. Clin. Invest. 122, 3990-4002 (2012).

16. Insel, P.A., Stengel, D., Ferry, N. \& Hanoune, J. Regulation of adenylate cyclase of human platelet membranes by forskolin. J. Biol. Chem. 257, 7485-7490 (1982).

17. Knowles, R.G., Palacios, M., Palmer, R.M. \& Moncada, S. Formation of nitric oxide from I-arginine in the central nervous system: a transduction mechanism for stimulation of the soluble guanylate cyclase. Proc. Natl. Acad. Sci. USA 86, 5159-5162 (1989).

18. Wechsler, J. et al. Isoforms of cyclic nucleotide phosphodiesterase PDE3A in cardiac myocytes. J. Biol. Chem. 277, 38072-38078 (2002).

19. Vandeput, F. et al. Selective regulation of cyclic nucleotide phosphodiesterase PDE3A isoforms. Proc. Natl. Acad. Sci. USA 110, 19778-19783 (2013).

20. Choi, Y.H. et al. Identification of a novel isoform of the cyclic-nucleotide phosphodiesterase PDE3A expressed in vascular smooth-muscle myocytes. Biochem. J. 353, 41-50 (2001).

21. Cone, J. et al. Comparison of the effects of cilostazol and milrinone on intracellular cAMP levels and cellular function in platelets and cardiac cells. J. Cardiovasc. Pharmacol. 34, 497-504 (1999).

22. Dunkerley, H.A. et al. Reduced phosphodiesterase 3 activity and phosphodiesterase 3 A level in synthetic vascular smooth muscle cells: implications for use of phosphodiesterase 3 inhibitors in cardiovascular tissues. Mol. Pharmacol. 61, 1033-1040 (2002).

23. Linglart, A. et al. Recurrent PRKAR1A mutation in acrodysostosis with hormone resistance. N. Engl. J. Med. 364, 2218-2226 (2011).

24. Salem, H.K. \& Thiemermann, C. Mesenchymal stromal cells: current understanding and clinical status. Stem Cells 28, 585-596 (2010).

25. Begum, N., Hockman, S. \& Manganiello, V.C. Phosphodiesterase 3A (PDE3A) deletion suppresses proliferation of cultured murine vascular smooth muscle cells (VSMCs) via inhibition of mitogen-activated protein kinase (MAPK) signaling and alterations in critical cell cycle regulatory proteins. J. Biol. Chem. 286 , 26238-26249 (2011)

26. Schuster, H. et al. Autosomal dominant hypertension and brachydactyly in a Turkish kindred resembles essential hypertension. Hypertension 28, 1085-1092 (1996).
27. Hunter, R.W., Mackintosh, C. \& Hers, I. Protein kinase C-mediated phosphorylation and activation of PDE3A regulate CAMP levels in human platelets. J. Biol. Chem. 284, 12339-12348 (2009).

28. Pozuelo Rubio, M., Campbell, D.G., Morrice, N.A. \& Mackintosh, C. Phosphodiesterase $3 \mathrm{~A}$ binds to $14-3-3$ proteins in response to PMA-induced phosphorylation of Ser428. Biochem. J. 392, 163-172 (2005).

29. Castagna, M. et al. Direct activation of calcium-activated, phospholipid-dependent protein kinase by tumor-promoting phorbol esters. J. Biol. Chem. 257, 7847-7851 (1982).

30. Graves, L.M. et al. Protein kinase A antagonizes platelet-derived growth factorinduced signaling by mitogen-activated protein kinase in human arterial smooth muscle cells. Proc. Natl. Acad. Sci. USA 90, 10300-10304 (1993).

31. Zhao, H., Guan, Q., Smith, C.J. \& Quilley, J. Increased phosphodiesterase 3A/4B expression after angioplasty and the effect on VASP phosphorylation. Eur. J. Pharmacol. 590, 29-35 (2008).

32. Song, G.J., Fiaschi-Taesch, N. \& Bisello, A. Endogenous parathyroid hormonerelated protein regulates the expression of PTH type 1 receptor and proliferation of vascular smooth muscle cells. Mol. Endocrinol. 23, 1681-1690 (2009).

33. Hundsrucker, C. et al. Glycogen synthase kinase $3 \beta$ interaction protein functions as an A-kinase anchoring protein. J. Biol. Chem. 285, 5507-5521 (2010).

34. Beca, $\mathrm{S}$. et al. Phosphodiesterase type $3 \mathrm{~A}$ regulates basal myocardial contractility through interacting with sarcoplasmic reticulum calcium ATPase type 2a signaling complexes in mouse heart. Circ. Res. 112, 289-297 (2013).

35. Lygren, B. et al. AKAP complex regulates $\mathrm{Ca}^{2+}$ re-uptake into heart sarcoplasmic reticulum. EMBO Rep. 8, 1061-1067 (2007).

36. Ahmad, F. et al. Regulation of SERCA2 activity by PDE3A in human myocardium phosphorylation-dependent interaction of PDE3A1 with SERCA2. J. Biol. Chem 290, 6763-6776 (2015)

37. Bassler, D., Kreutzer, K., McNamara, P. \& Kirpalani, H. Milrinone for persistent pulmonary hypertension of the newborn. Cochrane Database Syst. Rev. CD007802 (2010)

38. Pfitzer, G. Invited review: regulation of myosin phosphorylation in smooth muscle J. Appl. Physiol. 91, 497-503 (2001).

39. Klopocki, E et al. Deletion and point mutations of PTHLH cause brachydactyly type E. Am. J. Hum. Genet. 86, 434-439 (2010).

40. Bastepe, M. et al. Stimulatory G protein directly regulates hypertrophic differentiation of growth plate cartilage in vivo. Proc. Natl. Acad. Sci. USA 101, 14794-14799 (2004).

41. Chilco, P.J., Leopold, V. \& Zajac, J.D. Differential regulation of the parathyroid hormone-related protein gene P1 and P3 promoters by cAMP. Mol. Cell. Endocrinol. 138, 173-184 (1998).

\section{Philipp G Maass $1,2,39,40$, Atakan Aydin ${ }^{1,2,40}$, Friedrich C Luft ${ }^{1-3,40}$, Carolin Schächterle ${ }^{2,40}$, Anja Weise Sigmar Stricker ${ }^{5,6}$, Carsten Lindschau ${ }^{7,8}$, Martin Vaegler ${ }^{1,9}$, Fatimunnisa Qadri ${ }^{1,2}$, Hakan R Toka ${ }^{10,11}$, Herbert Schulz ${ }^{2,12}$, Peter M Krawitz ${ }^{5,13,14}$, Dmitri Parkhomchuk ${ }^{5,13,14}$, Jochen Hecht ${ }^{5,14}$, Irene Hollfinger ${ }^{2}$, Yvette Wefeld-Neuenfeld ${ }^{2}$, Eireen Bartels-Klein ${ }^{2}$, Astrid Mühl' ${ }^{2}$, Martin Kannn ${ }^{15,16}$, Herbert Schuster ${ }^{17}$, David Chitayat ${ }^{18,19}$, Martin G Bialer ${ }^{20,21}$, Thomas F Wienker, ${ }^{5,22}$, Jürg Ott ${ }^{23,24}$, Katharina Rittscher ${ }^{4}$, Thomas Liehr ${ }^{4}$, Jens Jordan ${ }^{25}$, Ghislaine Plessis ${ }^{26}$, Jens Tank ${ }^{25}$, Knut Mai ${ }^{1}$, Ramin Naraghi ${ }^{27}$, Russell Hodge ${ }^{2}$, Maxwell Hopp ${ }^{28}$, Lars O Hattenbach ${ }^{29}$, Andreas Busjahn ${ }^{30}$, Anita Rauch ${ }^{31}$, Fabrice Vandeput ${ }^{32-34}$, Maolian Gong ${ }^{1,2}$, Franz Rüschendorf ${ }^{2}$, Norbert Hübner ${ }^{2,35,36}$, Hermann Haller ${ }^{7}$, Stefan Mundlos ${ }^{5,13,14}$ Nihat Bilginturan ${ }^{37}$, Matthew A Movsesian ${ }^{32-34}$, Enno Klussmann ${ }^{2,35}$, Okan Toka ${ }^{38}$ \& Sylvia Bähring ${ }^{1,2,40}$}

\footnotetext{
${ }^{1}$ Experimental and Clinical Research Center (ECRC), a joint cooperation between the Charité Medical Faculty and the Max Delbrück Center for Molecular Medicine in the Helmholtz Association (MDC), Berlin, Germany. ${ }^{2}$ Max Delbrück Center for Molecular Medicine in the Helmholtz Association (MDC), Berlin, Germany. ${ }^{3}$ Division of Clinical Pharmacology, Department of Medicine, Vanderbilt University School of Medicine, Nashville, Tennessee, USA. ${ }^{4}$ Institute of Human Genetics, Jena University Hospital, Friedrich Schiller University, Jena, Germany. ${ }^{5}$ Max Planck Institute for Molecular Genetics, Berlin, Germany. ${ }^{6}$ Institute for Chemistry and Biochemistry, Freie Universität Berlin, Berlin, Germany. ${ }^{7}$ Department of Nephrology, Hannover University Medical School, Hannover, Germany. ${ }^{8}$ Staatliche Technikerschule Berlin, Berlin, Germany. ${ }^{9}$ Department of Urology, Laboratory of Tissue Engineering, Eberhard Karls University Tübingen, Tübingen, Germany. ${ }^{10}$ Division of Nephrology and Hypertension, Eastern Virginia Medical School, Norfolk, Virginia, USA. ${ }^{11}$ Division of Nephrology, Brigham and Women's Hospital, Boston, Massachusetts, USA. ${ }^{12}$ Cologne Center for Genomics (CCG), University of Cologne, Cologne, Germany. ${ }^{13}$ Institute for Medical Genetics and Human Genetics, Charité Universitätsmedizin Berlin, Berlin, Germany. ${ }^{14}$ Berlin Brandenburg Center for Regenerative Therapies (BCRT), Charité Universitätsmedizin Berlin, Berlin, Germany. ${ }^{15}$ Department II of Medicine, University of Cologne, Cologne, Germany. ${ }^{16}$ Center for Molecular Medicine Cologne, University of Cologne, Cologne, Germany. ${ }^{17}$ INFOGEN, Berlin, Germany. ${ }^{18}$ Division of Clinical and Metabolic Genetics, The Hospital for Sick Children, University of Toronto, Toronto, Ontario, Canada. ${ }^{19}$ Prenatal Diagnosis and Medical Genetics Program, Department of Obstetrics and Gynecology, Mount Sinai Hospital, University of Toronto, Toronto, Ontario, Canada. ${ }^{20}$ Division of Medical Genetics, North Shore/LIJ Health System, Manhasset, New York, USA. ${ }^{21}$ Department of Pediatrics, North Shore/LIJ Health System, Manhasset, New York, USA. ${ }^{22}$ Institute for Medical Biometry, Informatics and Epidemiology, University of Bonn, Bonn, Germany. ${ }^{23}$ Institute of Psychology, Chinese Academy of Sciences, Beijing, China. ${ }^{24}$ Statistical Genetics, Rockefeller University, New York, New York, USA. ${ }^{25}$ Institute of Clinical Pharmacology, Hannover Medical School, Hannover, Germany. ${ }^{26}$ Centre Hospitalier Universitaire de Caen, Cytogénétique Postnatale et Génétique Clinique, Caen, France. ${ }^{27}$ Department of Neurosurgery, Bundeswehrkrankenhaus UIm, Ulm, Germany. ${ }^{28}$ Department of Pediatrics, Griffith Base Hospital, Griffith, New South Wales, Australia. ${ }^{29}$ Department of Ophthalmology, Hospital Ludwigshafen, Ludwigshafen, Germany. ${ }^{30}$ HealthTwist, Berlin, Germany. ${ }^{31}$ Institute for Medical Genetics, University of Zurich, Zurich, Switzerland. ${ }^{32}$ Cardiology Section, Veterans Affairs Salt Lake City Health Care System, Salt Lake City, Utah, USA. ${ }^{33}$ Department of Internal Medicine, University of Utah, Salt Lake City, Utah, USA. ${ }^{34}$ Department of Pharmacology and Toxicology, University of Utah, Salt Lake City, Utah, USA. ${ }^{35}$ DZHK (German Centre for Cardiovascular Research), Berlin, Germany. ${ }^{36}$ Charité Universitätsmedizin, Berlin, Germany. ${ }^{37}$ Department of Pediatric Oncology, Hacettepe University, Ankara, Turkey. ${ }^{38}$ Department of Pediatric Cardiology, Children's Hospital, Friedrich Alexander University Erlangen, Erlangen, Germany. ${ }^{39}$ Present address: Department of Stem Cell and Regenerative Biology, Harvard University, Cambridge, Massachusetts, USA. ${ }^{40}$ These authors contributed equally to this work. Correspondence should be addressed to F.C.L. (friedrich.luft@charite.de).
} 


\section{ONLINE METHODS}

Study approval, human material. We studied six unrelated families. After approval by the ethics committee (Charité Medical Faculty, Berlin) and written informed consent, we obtained skin fibroblasts from six affected individuals (three male, three female) and eight controls (five male, three female) from the Turkish kindred; mean age was $37.7 \pm 0.4$ years. We extracted MSCs from two affected Turkish males (VI/9, 17 years old; VI/17, 12 years old) and from two unaffected, non-related controls (male, 15 years old; female, 59 years old).

Whole-genome sequencing, Sanger sequencing and SNP analysis. Complete Genomics performed whole-genome sequencing of three affected patients and one control from a Turkish nuclear family ${ }^{42}$. Whole-genomic DNA was extracted from EDTA-blood using standard procedures. A mean per-sample depth of coverage of 57.39 $\times$ was reached. After bioinformatic analysis, data were further analyzed with CGA tools. Among the Complete Genomicsannotated variants in coding genes within the linkage interval, only the $P D E 3 A$ missense mutation resulting in p.Thr445Asn was detected in three affected patients of the Turkish nuclear family. Numerous insertion, deletion or substitution events within the linkage region, indicating micromutations at the putative inversion breakpoints, were found. After excluding annotated SNPs, we selected heterozygous variants that were not detectable in the unaffected control or in the hg19 genome assembly. These variants were further analyzed in a larger cohort (seven affected and seven unaffected individuals; Supplementary Table 1). Sanger resequencing of the seven affected individuals and seven controls identified only polymorphisms. After identification of the putative inversion breakpoint regions by interphase FISH, the genomic regions with Complete Genomics sequencing reads were further analyzed in the CGI browser (data not shown). Reads for the genomic region of interest were extracted from the Complete Genomics-annotated variants and visualized. None of the variants corresponded to the inversion breakpoints.

An Illumina HiSeq 2000 was used to generate 101-bp paired-end reads, and $374 \mathrm{~Gb}$ of raw sequence data were mapped to the human reference GRCh37.3 with Novoalign, yielding an average coverage of more than 100-fold. Singlenucleotide variants and small indels were detected with the Genome Analysis Toolkit (GATK) ${ }^{43}$, annotated with ANNOVAR ${ }^{44}$ and analyzed on GeneTalk ${ }^{45}$. Structural variants were detected with BreakDancer ${ }^{46}$, and the previously reported inversion was also analyzed as described ${ }^{47}$ but did not show any inversion breakpoints.

The primers for exon 4 of $P D E 3 A$ used to detect the mutations are shown in Supplementary Table 3. After PCR amplification, amplicons were sequenced using BigDye Terminator Cycle Sequencing Kit v1.1 (Applied Biosystems). Analysis was performed on a 3130xl Genetic Analyzer (Applied Biosystems) using Gene Mapper Software Version 4.0. SeqMan software (Lasergene, Version 10.0; DNAStar) was used to evaluate the traces.

Whole-mount RNA in situ hybridization. In situ hybridizations were carried out using digoxygenin-UTP-labeled sense and antisense riboprobes for mouse Pde3a (coding sequence probe, chr. 6: 141,459,712-141,471,161 bp; 3' UTR probe, chr. 6: 141,498,908-141,499,348; UCSC Genome Browser, mm10) according to standard protocols. The experiments were approved by the competent local authority in Berlin (LaGeSo).

HeLa cells and chondrogenic induction of fibroblasts. HeLa cells were ordered from the American Type Culture Collection (ATCC) and grown in DMEM supplemented with $10 \%$ FCS, $100 \mathrm{U} / \mathrm{ml}$ penicillin and $100 \mu \mathrm{g} / \mathrm{ml}$ streptomycin. The cells were frequently tested for mycoplasma contamination. Patient- and control-derived fibroblasts were cultured in Eagle's MEM 199 supplemented with $10 \%$ FCS, $100 \mathrm{U} / \mathrm{ml}$ penicillin and $100 \mu \mathrm{g} / \mathrm{ml}$ streptomycin. The chondrogenic induction of fibroblasts was carried out in pellet cultures with DMEM supplemented with $10 \% \mathrm{FCS}, 100 \mathrm{U} / \mathrm{ml}$ penicillin, $100 \mu \mathrm{g} / \mathrm{ml}$ streptomycin, $1 \times$ ITS-X (Life Technologies), $10 \mathrm{ng} / \mathrm{ml}$ human transforming growth factor (TGF)- $\beta 1$ (R\&D Systems), $500 \mathrm{ng} / \mathrm{ml}$ recombinant human insulin-like growth factor (IGF)-1 (R\&D Systems), $50 \mu \mathrm{M}$ L-ascorbic-2-phosphate (Sigma) and L-glutamine (PAA) for 21-28 d. Fibroblasts were fixed for $10 \mathrm{~min}$ with $4 \%$ paraformaldehyde (Sigma) and stained in 1\% Alcian blue (Chroma; 3\% acetic acid) for $30 \mathrm{~min}$ or in $6 \%$ Safranin O (Sigma; in distilled water) for $2 \mathrm{~min}$. Before microscopic documentation, cells were washed twice with $90 \%$ ethanol.
Expression plasmids. Each of the six $P D E 3 A$ mutations were introduced into the full-length cDNA clone for wild-type PDE3A (NM_000921; Origene, SC300151) by in vitro mutagenesis according to the manufacturer's protocol (QuikChange II XL Site-Directed Mutagenesis kit, Ambion Technologies). The recommended PCR extension times were doubled to amplify the large plasmids. In transfected HeLa cells, PDE3A1 translation started at amino acid 146 and PDE3A2 translation was initiated from amino acid 300; PDE3A3 translation could be started from position 484, but this isoform was not expressed in transiently transfected HeLa cells. The mutation encoding p.Thr445Asn was also introduced into expression plasmids encoding Flag-tagged PDE3A1 and PDE3A2 that we had previously characterized ${ }^{19}$. Recombinant Flag-tagged PDE3A1 and PDE3A2 and their mutated versions with alanine substitutions at positions Ser428, Ser438, Ser439 and Thr440 were used in Michaelis-Menten kinetics and $\mathrm{IC}_{50}$ measurements. Sanger sequencing validated the integrity of the entire PDE3A ORF of each plasmid.

cAMP and cGMP enzyme immunoassays. HeLa cells $\left(4.5 \times 10^{5}\right)$ were seeded in a $6-\mathrm{cm}$ petri dish. After overnight incubation, cells were transfected with 1 pmol of each full-length PDE3A expression plasmid and $20 \mu \mathrm{l}$ of FuGENE Xtreme Gene HP (Roche) for $48 \mathrm{~h}$. Before cell lysis, stimulation with $20 \mu \mathrm{M}$ forskolin (Sigma) or $10 \mu \mathrm{M} \mathrm{L}$-arginine (Sigma) was carried out for $30 \mathrm{~min}$. Cell lysates were used for cAMP and cGMP assays (R\&D Systems) that were performed according to the user's manual. BCA-quantified total protein measurements for the cell lysates were used for normalization.

CFSE proliferation assays. HeLa cells $\left(4 \times 10^{5}\right)$ or VSMCs $\left(2 \times 10^{5}\right)$, cultured in $20 \%$ FCS, were labeled with CFSE $\left(10 \mu \mathrm{M}\right.$ per $1 \times 10^{6}$ cells $)$ for each time point according to the manufacturer's recommendation (Life Technologies). CFSE-labeled cells were seeded 12-24 h before transfection with $500 \mathrm{fmol}$ of each expression plasmid and $8 \mu$ l of FuGENE Xtreme Gene HP. The first flow cytometric measurement of CFSE-labeled cells was performed after overnight incubation and before transfection at time point $0 \mathrm{~h}$. Every $24 \mathrm{~h}$ after transfection, cells were analyzed on a BD FACSCalibur flow cytometer. Cell numbers were determined by normalizing the levels of wild-type PDE3A and the six PDE3A mutants to endogenous PDE3A levels of control HeLa cells transfected with empty vector. Differentiation of VSMCs was performed for 14-21 d. VSMC differentiation medium was changed after CFSE labeling to DMEM supplemented with $20 \%$ FCS and $1 \mathrm{ng} / \mathrm{ml}$ TGF- $\beta 1$. Proliferation of VSMCs was normalized to cell numbers at the starting point of $0 \mathrm{~h}$.

Immunoprecipitation of Flag-tagged wild-type and Thr445Asn PDE3A1, and PDE3A2 Michaelis-Menten kinetics and $\mathrm{IC}_{\mathbf{5 0}}$ measurements. Transfected HeLa cells $\left(2 \times 10^{6}\right)$ transiently expressing Flag-tagged versions of PDE3A1 or PDE3A2 were scraped from two $10-\mathrm{cm}$ cell culture dishes in $1 \mathrm{ml}$ of PBS. Before cell collection, $20 \mu \mathrm{M}$ forskolin (Sigma) or DMSO (for controls) was used to stimulate cells for $30 \mathrm{~min}$. Quick centrifugation $\left(275 \mathrm{~g}\right.$ for $5 \mathrm{~min}$ at $4{ }^{\circ} \mathrm{C}$ ) pelleted cells for lysis in $100 \mu$ of RIPA buffer (50 mM Tris, pH 7.8, 10\% glycerol, $150 \mathrm{mM} \mathrm{NaCl}, 1 \%$ Triton X, $0.025 \%$ sodium deoxycholate and $1 \mathrm{mM}$ EDTA) supplemented with protease and phosphatase inhibitors (Complete and PhosSTOP, Roche Diagnostics). Cells were incubated for $10 \mathrm{~min}$ on ice and subsequently centrifuged $\left(21,250 \mathrm{~g}\right.$ for $15 \mathrm{~min}$ at $4{ }^{\circ} \mathrm{C}$; Eppendorf). ANTI-FLAG M2 Magnetic Beads (Sigma-Aldrich) were thoroughly resuspended: $80 \mu 1$ of beads for each lysate were washed five times in Tris-buffered saline (TBS), mixed thoroughly and placed on a magnetic separator to remove washing buffer. Equilibrated beads were loaded with equal amounts of protein lysate (according to BCA quantification) and incubated overnight at $4{ }^{\circ} \mathrm{C}$ on a rotating device. After discarding the supernatant, the beads were washed three times with TBS supplemented with protease and phosphatase inhibitors. Washed beads bound to Flag-tagged PDE3A1 or PDE3A2 proteins were resuspended in $800 \mu \mathrm{l}$ of incubation buffer ( $10 \mathrm{mM}$ Tris, $\mathrm{pH} 8.0$ and $10 \mathrm{mM} \mathrm{MgCl}_{2}$ ), and $10 \%$ were used for each cAMP hydrolysis assay. Incubation of purified beads-bound enzymes with increasing concentrations of cAMP or with the milrinone or cGMP inhibitor were carried out at $30^{\circ} \mathrm{C}$ and $1,100 \mathrm{rpm}$ for $30 \mathrm{~min}$ in a thermomixer. In the inhibitor assays, another incubation at $30^{\circ} \mathrm{C}$ and $1,100 \mathrm{rpm}$ for $30 \mathrm{~min}$ followed after the addition of $250 \mathrm{nM}$ cAMP as substrate. Tubes were placed in a magnetic separator, and the supernatant was transferred to a new tube filled with neutralization buffer (cAMP Direct Immunoassay kit, Biovision). 
Further steps were performed as described in the instruction manual. The magnetic beads were resuspended in $10 \times$ SDS loading buffer $(50 \mathrm{mM}$ Tris, $\mathrm{pH}$ $6.8,1.6 \%$ SDS, $4 \%$ glycerol, $0.6 \% \beta$-mercaptoethanol and bromophenol blue) and incubated for $5 \mathrm{~min}$ at $95^{\circ} \mathrm{C}$. Immunoblotting of each sample was used to validate equal enzyme loading for each condition. The Flag-tagged PDE3A1 and PDE3A2 proteins were detected using antibodies to PDE3A (A302-740A, Bethyl Laboratories) and PDE3A phosphorylated at Ser438 (S442B, University of Dundee). Signals were visualized using Immobilon Western (Merck Millipore) and the Odyssey Fc Dual-Mode Imaging System (Li-Cor). $K_{\mathrm{M}}$ and $\mathrm{IC}_{50}$ values were calculated using GraphPad Prism software (version 5.01). Instead of inaccurate linear regression, we used nonlinear regression analysis with OLS (ordinary least-squares) curve fitting and a Hill slope of -1 or 1 , respectively, to determine the $\mathrm{IC}_{50}$ values and Michaelis-Menten kinetics shown in Figures 3 and $\mathbf{5}$ (see also Supplementary Figs. 4 and 5). According to GraphPad tutorials, the calculated Michaelis-Menten values were transformed to generate double-reciprocal Lineweaver-Burke plots (Supplementary Fig. 4d-k).

Mesenchymal stromal cell extraction, cultivation, characterization and differentiation. MSCs for two affected individuals and two unaffected, related controls were extracted from peripheral blood in heparin sulfate ${ }^{48}$. We determined that the frequency was $6 \%$ for successful MSC extraction from peripheral blood. Because the two controls were too old ( $>65$ years), their MSCs did not demonstrate multilineage potential. The documented MSC controls used were extracted from the lipoaspirates of unaffected, unrelated family members. Fat biopsies were washed with $1 \%$ AntiAnti (Life Technologies) in PBS. Larger tissue was mechanically destructed using a scalpel, and the resulting suspension was washed with $1 \%$ AntiAnti in PBS over a $100-\mu \mathrm{m}$ cell strainer. Subsequent PBS washing removed erythrocytes and blood. Homogenization was carried out by digestion with collagenase I ( $0.1 \%$ in PBS) with $1 \%$ BSA for $60 \mathrm{~min}$ at $37^{\circ} \mathrm{C}$. After $10 \mathrm{~min}$ of incubation with $15 \mathrm{ml}$ of DMEM (low glucose), the adipocytes on the surface were removed. The remaining cell suspension was filtered again using a $100-\mu \mathrm{m}$ cell strainer and centrifuged for $10 \mathrm{~min}$ at room temperature at $250 \mathrm{~g}$. After discarding the supernatant, the cell pellet was washed with $15 \mathrm{ml}$ of DMEM and centrifuged again for $5 \mathrm{~min}$. After centrifugation, the cells were resuspended in DMEM (low glucose) supplemented with $100 \mathrm{U} / \mathrm{ml}$ penicillin, $100 \mu \mathrm{g} / \mathrm{ml}$ streptomycin, $2 \mathrm{IU} / \mathrm{ml}$ heparin sulfate, $5 \%$ human fresh-frozen plasma (FFP) and 5\% concentrated thrombocytes. Depending on the volume of the lipoaspirate available, one of the following cultures was set up: $1 \mathrm{ml}$ of cell suspension in a $25-\mathrm{cm}^{2}$ dish, up to $3 \mathrm{ml}$ of cell suspension in a $75-\mathrm{cm}^{2}$ dish or more than $3 \mathrm{ml}$ of cell suspension in a $150-\mathrm{cm}^{2}$ dish. After $24 \mathrm{~h}$, the cells were washed five times with PBS, and new medium was added. After 3-4 d, stretched-out, fibroblast-like cells were observed. The splitting ratio for subconfluent MSCs was 1:5 or 1:6. Passages up to passage 8 were used in the described experiments. Upon FACS characterization for $\mathrm{CD}_{105^{+}}, \mathrm{CD}^{2} 0^{+}, \mathrm{CD}_{73}{ }^{+}, \mathrm{HLA}_{-} \mathrm{ABC}^{+}, \mathrm{CD} 31^{-}, \mathrm{CD} 34^{-}, \mathrm{CD} 45^{-}$ and HLA-DR ${ }^{-}$cells, MSCs were differentiated. The adipogenic differentiation medium constituted DMEM ( $1 \mathrm{~g} / \mathrm{l}$ glucose), $1 \mathrm{IU} / \mathrm{ml}$ heparin sulfate, $1 \%$ concentrated thrombocytes, $5 \% \mathrm{FFP}, 100 \mathrm{U} / \mathrm{ml}$ penicillin, $100 \mu \mathrm{g} / \mathrm{ml}$ streptomycin, $1 \mu \mathrm{M}$ dexamethasone, $100 \mu \mathrm{M}$ L-ascorbic-2-phosphate, $60 \mu \mathrm{M}$ indomethacin, $0.5 \mu \mathrm{M}$ IBMX and $10 \mu \mathrm{M}$ insulin (Sigma). After 2-5 weeks of differentiation, cells were fixed with steams of $40 \%$ formalin for $10 \mathrm{~min}$, washed twice with distilled water and stained for $3 \mathrm{~min}$ in $1 \%$ oil red. The amount of time needed for differentiation was dependent on the presence of fatty vacuoles. Osteogenic differentiation was performed within 2 weeks. The differentiation medium for the first week contained DMEM ( $1 \mathrm{~g} / \mathrm{l}$ glucose), $1 \mathrm{IU} / \mathrm{ml}$ heparin sulfate, $1 \%$ concentrated thrombocytes, $5 \% \mathrm{FFP}, 100 \mathrm{U} / \mathrm{ml}$ penicillin, $100 \mu \mathrm{g} / \mathrm{ml}$ streptomycin, $10 \mathrm{nM}$ dexamethasone and $100 \mu \mathrm{M} \mathrm{L}$-ascorbic-2-phosphate. For the medium for the second week, $100 \mathrm{ng} / \mathrm{ml}$ recombinant human bone morphogenetic protein 2 (BMP2; R\&D Systems) and $10 \mathrm{mM} \beta$-glycerophosphate were also added. After a PBS wash, cells were fixed with ice-cold methanol, air dried and stained with $0.5 \%$ Alizarin red for $30 \mathrm{~s}$. After a 15-min PBS wash, calcium precipitates were microscopically documented. Chondrogenic differentiation was performed in micromass pellet cultures for up to 3 weeks. DMEM ( $4.5 \mathrm{~g} / 1$ glucose) was supplemented with $1 \mathrm{IU} / \mathrm{ml}$ heparin sulfate, 5\% FFP, $1 \times \mathrm{ITS}-\mathrm{X}$, $100 \mathrm{U} / \mathrm{ml}$ penicillin, $100 \mu \mathrm{g} / \mathrm{ml}$ streptomycin, $100 \mathrm{nM}$ dexamethasone, $50 \mu \mathrm{M}$ L-ascorbic-2-phosphate, $100 \mathrm{ng} / \mu \mathrm{l}$ recombinant human IGF-1, $10 \mathrm{ng} / \mathrm{ml}$ recombinant human TGF- $\beta 1$ and $1 \mathrm{mM}$ sodium pyruvate. Chondrogenic pellets were fixed overnight with $4 \%$ paraformaldehyde, and paraffin-embedded tissue was sectioned for toluidin blue staining. The smooth muscle differentiation medium contained DMEM ( $1 \mathrm{~g} / \mathrm{l}$ glucose), $1 \mathrm{IU} / \mathrm{ml}$ heparin sulfate, $5 \%$ FFP, $100 \mathrm{U} / \mathrm{ml}$ penicillin, $100 \mu \mathrm{g} / \mathrm{ml}$ streptomycin and $1 \mathrm{ng} / \mathrm{ml}$ recombinant human TGF- $\beta 1$. The medium was exchanged every $2 \mathrm{~d}$ for a period of $21 \mathrm{~d}$.

FACS. FACS staining of extracellular surface markers was carried out according to standard procedures on a BD FACSCalibur. The following antibodies were used: antibody to CD105 (MHCD10504, Invitrogen), antibody to CD90 (555595, BD Biosciences), antibody to CD73 (550257, BD Biosciences), antibody to HLAABC (555552, BD Biosciences), antibody to CD31 (555445, BD Biosciences), antibody to CD34 (555821, BD Biosciences), antibody to CD45 (555482, BD Biosciences) and antibody to HLA-DR (555561, BD Biosciences).

Antibodies and immunoblotting. After $48 \mathrm{~h}$ of transfection, HeLa cells were stimulated with either $20 \mu \mathrm{M}$ forskolin or $100 \mathrm{ng} / \mathrm{ml}$ PMA for $30 \mathrm{~min}$. Immunoblotting was performed according to standard protocols with $20 \mu \mathrm{g}$ of total protein quantified by the BCA test (Thermo Scientific). Antibodies to PDE3A phosphorylated at Ser428 (S446B) and Ser438 (S442B) were ordered from the University of Dundee. C. MacKintosh validated the specificity of the antibodies and their usage in several projects ${ }^{27,28}$. The following antibodies were also used: antibody to PDE3A (A302-740A, Bethyl Laboratories), antibody to PTHLH (ab41438, Abcam), antibody to PTHLH (ab115488, Abcam), antibody to $\beta$-tubulin (sc-9104, Santa Cruz Biotechnology), antibody to VASP phosphorylated at Ser157 (ab47268, Abcam), antibody to VASP (sc-13975, Santa Cruz Biotechnology), antibody to SMA $\alpha$ (ab8211, Abcam), antibody to calponin (ab700, Abcam), antibody to SM22 $\alpha$ (ab10135, Abcam) and antibody to Renilla luciferase (MAB4400, Millipore). Competitors for the antibodies recognizing phosphorylated proteins were the appropriate peptides from Santa Cruz Biotechnology and the University of Dundee. Detection of Renilla luciferase, cotransfected into HeLa cells, indicated equal transfection and expression conditions. Blot signals were determined by horseradish peroxidase (HRP)-mediated chemiluminescence (SuperSignal West Pico, Thermo Scientific) and visualized in a PeqLab, Chemi-Smart 5000.

Immunofluorescence. For semiquantitative confocal microscopy to investigate proteins and their phosphorylation states, MSCs, VSMCs or transiently transfected HeLa cells were grown on glass coverslips. After one PBS wash, cells were fixed with fresh $4 \%$ paraformaldehyde for $10 \mathrm{~min}$ at room temperature and permeabilized with $80 \%$ methanol for $20 \mathrm{~min}$ at $-20^{\circ} \mathrm{C}$. After blocking with $2 \%$ BSA in PBS and incubation with primary antibody at the appropriate dilution (see the antibodies listed in "Antibodies and immunoblotting"), staining was accomplished with Alexa Fluor 488-coupled secondary antibodies. Visualization was performed with a Bio-Rad MRC1024 attached to a Nikon Diaphot inverted microscope using the 488-nm line from an argon/krypton laser with a kalman filter of 3 . After detection, the pseudocolors used indicated expression levels from black (low) to red (high).

Luciferase reporter assays. HeLa cells $\left(5 \times 10^{4}\right)$ were seeded $12-24 \mathrm{~h}$ before transfection. Equal molarities were used for transient transfection to compensate for the diverse plasmid sizes; $75 \mathrm{fmol}$ each of the CRE-luciferase construct (pGL4.29, Promega) and the full-length wild-type PDE3A or full-length mutant constructs were cotransfected into cells with FuGENE Extreme Gene HP, according to the manufacturer's recommendations. The CRE-luciferase construct harbored a cAMP-responsive element that drove transcription of the luciferase gene. Thus, the measured luciferase activity was dependent on cellular cAMP levels. pRL-TK (12.5 ng; Promega) was added to control for transfection efficiency. After $48 \mathrm{~h}$ of transfection, either total RNA was prepared or cell lysates were analyzed using the Dual-Glo Assay (Promega) in a Berthold luminometer. Four hours before cell lysis, forskolin was added ${ }^{23}$. Forskolin activates adenylate cyclase for cAMP production. Forskolinmediated increased cAMP levels caused luciferase upregulation. L-arginine enhances guanylate cyclase activity for cGMP production and was added for 10-15 min before cell lysis ${ }^{17}$. Because cGMP inhibits PDE-mediated cAMP hydrolysis in a competitive manner, luciferase transcription was upregulated. In all functional in vitro experiments, DMSO served as the control. 
Peptide arrays, PKA phosphorylation of SPOT-synthesized peptides and immunoblotting. Peptide spots were generated by automatic SPOT synthesis using the Intavis ResPep-SL device (Intavis) as described previously33,49-51. Fmoc-protected amino acids were purchased from Intavis; phosphoserine (Fmoc-Ser(PO-(OBzl)OH)-OH) was from Novabiochem (Merck Millipore). Derivatized cellulose membranes (amino-modified acid-stable cellulose membranes with PEG spacer) were also purchased from Intavis.

The membranes were briefly soaked in ethanol, blocked in blocking buffer (5\% milk in TBST (10 mM Tris- $\mathrm{HCl}, 150 \mathrm{mM} \mathrm{NaCl}$ and $0.05 \%$ Tween-20, $\mathrm{pH}$ 7.4) for $3 \mathrm{~h}$ at room temperature and subsequently washed twice with incubation buffer ( $50 \mathrm{mM}$ Tris- $\mathrm{HCl}, 5 \mathrm{mM} \mathrm{MgCl} 2$ and $100 \mu \mathrm{M} \mathrm{ATP}$ ). For phosphorylation of the peptides by PKA, His-tagged recombinant catalytic subunits (vector pET46) were purified from Escherichia coli (strain Rosetta D3) as described for RII $\alpha$ subunits ${ }^{52}$. The peptide spots were incubated with the recombinant catalytic subunits $(1 \mathrm{nM})$ in incubation buffer for $1 \mathrm{~h}$ at $30^{\circ} \mathrm{C}$. The membranes were washed three times with TBST. Phosphorylated serine was detected by incubation with PKA phosphosubstrate antibody recognizing the consensus site RRXp(S/T) (9624, Cell Signaling Technology) in blocking buffer overnight at $4{ }^{\circ} \mathrm{C}^{53}$. The membranes were washed three times with TBST. Thereafter, HRP-coupled donkey anti-rabbit secondary antibody (711-036153, Jackson ImmunoResearch) was added (for $3 \mathrm{~h}$ at room temperature), the membranes were washed three times with TBST and an enhanced chemiluminescence reaction was carried out using Immobilon Western substrate (Merck Millipore). Signals were visualized with the Odyssey FC device.

Pyrosequencing. Pyrosequencing was carried out according to standard procedures ${ }^{54}$. After cDNA synthesis of total RNA, the oligonucleotides listed in Supplementary Table 3 were used.

TaqMan expression analysis. RNA was prepared using TRIzol Reagent (Life Technologies) and reverse transcribed with the First-Strand cDNA Synthesis kit (Fermentas). Quantitative RT-PCR was performed according to standard protocols on an ABI 7500. TaqMan gene expression assays were used for PTHLH and GAPDH amplification (Life Technologies) ${ }^{55}$. Expression was quantified by applying the $\Delta \Delta C_{t}$ method.

Statistics. The differentiation into smooth muscle cells and the functional in vitro experiments were reproduced 2-6 times. Numbers $(n)$ of experiments are mentioned in the figure legends. Significance was determined by non-parametrical Wilcoxon-Mann-Whitney rank-sum testing or two-tailed Student's $t$ test $\left({ }^{* *} P<0.001,{ }^{* *} P<0.01,{ }^{\star} P<0.05\right)$. Scatterplots show the mean, and $\mathrm{T}$ bars indicate s.e.m.

42. Drmanac, R. et al. Human genome sequencing using unchained base reads on self-assembling DNA nanoarrays. Science 327, 78-81 (2010).

43. McKenna, A. et al. The Genome Analysis Toolkit: a MapReduce framework for analyzing next-generation DNA sequencing data. Genome Res. 20, 1297-1303 (2010).

44. Wang, K., Li, M. \& Hakonarson, H. ANNOVAR: functional annotation of genetic variants from high-throughput sequencing data. Nucleic Acids Res. 38, e164 (2010).

45. Kamphans, T. \& Krawitz, P.M. GeneTalk: an expert exchange platform for assessing rare sequence variants in personal genomes. Bioinformatics 28, 2515-2516 (2012).

46. Chen, K. et al. BreakDancer: an algorithm for high-resolution mapping of genomic structural variation. Nat. Methods 6, 677-681 (2009).

47. Spielmann, M. et al. Homeotic arm-to-leg transformation associated with genomic rearrangements at the PITX1 locus. Am. J. Hum. Genet. 91, 629-635 (2012).

48. Hofmann, N.A., Reinisch, A. \& Strunk, D. Isolation and large scale expansion of adult human endothelial colony forming progenitor cells. J. Vis. Exp. 32, 1524 (2009).

49. Hundsrucker, C. et al. High-affinity AKAP7 $\delta$-protein kinase A interaction yields novel protein kinase A-anchoring disruptor peptides. Biochem. J. 396, 297-306 (2006).

50. Coin, I., Beyermann, M. \& Bienert, M. Solid-phase peptide synthesis: from standard procedures to the synthesis of difficult sequences. Nat. Protoc. 2, 3247-3256 (2007).

51. Stefan, E. et al. Compartmentalization of cAMP-dependent signaling by phosphodiesterase-4D is involved in the regulation of vasopressin-mediated water reabsorption in renal principal cells. J. Am. Soc. Nephrol. 18, 199-212 (2007).

52. Schäfer, G. et al. Highly functionalized terpyridines as competitive inhibitors of AKAP-PKA interactions. Angew. Chem. Int. Edn Engl. 52, 12187-12191 (2013).

53. Christian, F. et al. Small molecule AKAP-protein kinase A (PKA) interaction disruptors that activate PKA interfere with compartmentalized CAMP signaling in cardiac myocytes. J. Biol. Chem. 286, 9079-9096 (2011).

54. Aydin, A., Toliat, M.R., Bahring, S., Becker, C. \& Nurnberg, P. New universal primers facilitate Pyrosequencing. Electrophoresis 27, 394-397 (2006).

55. Maass, P.G. et al. A cis-regulatory site downregulates PTHLH in translocation $\mathrm{t}(8 ; 12)(\mathrm{q} 13 ; \mathrm{p} 11.2)$ and leads to Brachydactyly Type E. Hum. Mol. Genet. 19, 848-860 (2010). 